\title{
Editorial for the ISC'10 Scientific Sessions Proceedings
}

\author{
Thomas Ludwig
}

Published online: 6 May 2010

(C) Springer-Verlag 2010

The International Supercomputing Conference started in 1986 as the "Supercomputer Seminar" organized by Prof. Hans Meuer, then director of the computer center and professor for computer science at the Universität Mannheim (Germany). Since these early days the annual conference became a major international event in the HPC community. As the conference grew bigger, it moved from Mannheim over Heidelberg and Dresden to Hamburg. In 2010 the conference will celebrate its 25th birthday! About 2,000 attendees and more than 140 exhibitors from over 45 countries are expected to attend, turning ISC' 10 into a powerful and memorable event.

Over the years we decided to strengthen the scientific part of the conference and present selected talks on relevant research results in the field of HPC. These scientific sessions started in 2007 as a separate day preceding the conference. Slides and accompanying papers were made available via the conference web site. Now, the scientific sessions evolved into an integral part of the conference, being scheduled in the afternoon of the first day. The call for participation was issued in winter 2009 inviting researchers and developers to submit latest results of their work as full research papers to the program committee of the scientific sessions.

Altogether we received 25 submissions from authors all over the world. In a peer-reviewing process with an international committee we selected the best 13 papers for publication in this journal and for presentation during the scientific sessions of the conference.

T. Ludwig $(\bowtie)$

Deutsches Klimarechenzentrum GmbH (DKRZ),

Bundesstrasse 45a, 20146 Hamburg, Germany

e-mail: ludwig@dkrz.de
We are happy to announce that many fascinating aspects of HPC will be discussed during the scientific sessions. The accepted publications will cover the following research and development areas:

- Large scale applications on GPGPU architectures

- Compiler and library aspects with multi-core architectures

- Programming on petascale systems

- Advanced parallel applications

We are confident that the ISC participants will find this selection appealing and that the presentations will generate inspiring discussions in the audience.

As in the years before, two awards were granted to the best papers, which were selected by an independent award committee.

The ISC'10 award is assigned to the following paper:

- Ping Lai, Sayantan Sur, Dhabaleswar K. Panda: Designing Truly One-Sided MPI-2 RMA Intra-node Communication on Multi-core Systems

PRACE, the Partnership for Advanced Computing in Europe, awards a price to the best scientific paper by a European student or young scientist. This year's award is granted to:

- Klaus Iglberger and Ulrich Rüde: Massively Parallel Granular Flow Simulations with Non-Spherical Particles

The proceedings of the scientific sessions are published in this special issue of Springer's Journal "Computer ScienceResearch and Development". Thomas Ludwig (Universität Hamburg and German Climate Computing Centre) and Julian Kunkel (German Climate Computing Centre) acted as guest editors.

We would like to express our gratitude to all the colleagues who submitted papers to the ISC scientific sessions, 
as well as to the members of the program committee for their contribution in reviewing these papers and for compiling an attractive program for the conference's audience.

Hamburg, May 2010

Thomas Ludwig Julian Kunkel Hans Meuer

\section{Program committee members}

- Galen Gisler, University of Oslo, Norway

- Lutz Gross, University of Queensland, Australia

- Wolfgang Hiller, AWI for Polar \& Marine Research, Germany

- Sverre Jarp, CERN, Switzerland

- Julian Kunkel, German Climate Computing Centre, Germany

- Fang-Pang Lin, National Center for High-performance Computing (NCHC), Taiwan

- Thomas Ludwig, University of Hamburg and German Climate Computing Centre, Germany (Chair)

- Federico Massaioli, CASPUR, Italy

- Satoshi Matsuoka, Tokyo Institute of Technology, Japan

- Wolfgang E. Nagel, Technische Universität Dresden, Germany

- Stephan Olbrich, University of Hamburg, Germany
- Michael M. Resch, HLRS Stuttgart, Germany

- Erich Strohmaier, Lawrence Berkeley National Laboratory, USA

- Yuichi Tsujita, Kinki University, Japan

\section{External reviewers}

- Panos Adamidis

- Cihan Altinay

- Jörg Behrens

- Vince Boros

- Ali Cevahir

- Toshio Endo

- Joel Fenwick

- Lin Gao

- Daniel Hackenberg

- Moritz Hanke

- Thomas Jahns

- Guido Juckeland

- Stephan Kindermann

- Andreas Knüpfer

- Michael Kuhn

- Matthias Lieber

- Naoya Maruyama

- Mathis Rosenhauer

- Niklas Röber

- Wolfgang V. Walter 